\title{
Comportamiento del residuo de vidrio molido en cementos mezcla: Estudio comparativo con microsilice
}

\author{
Behavior of the ground glass waste in \\ blending cements: Comparative \\ study with microsilice.
}

Mónica Adriana Trezza ${ }^{1}$, Viviana Fátima Rahhal ${ }^{1}$,

\footnotetext{
${ }^{1}$ Facultad de Ingeniería (UNCPBA) y CIFICEN (UNCPBA-CICPBA-CONICET), Av. del Valle 5737, (B7400JWI) Olavarría, Argentina.

e-mail: mtrezza@ fio.unicen.edu.ar
}

\section{RESUMEN}

Por su alto contenido de sílice en estado amorfo, los vidrios en teoría pueden comportarse como materiales puzolánicos cuando se muelen finamente. Esto abre una interesante vía de reutilización de estos residuos en la industria del cemento portland (CP), sobre todo si se considera que los residuos sólidos urbanos (RSU) en Argentina contienen entre el 3 y $5 \%$ en peso de vidrio.

Este trabajo estudia el comportamiento de residuos de vidrio blanco molido, procedente de botellas en comparación al correspondiente a la microsílice (MS), de reconocida actividad. El objetivo de este trabajo es analizar las diferencias estructurales, composición mineralógica, tamaño y forma de partícula de la MS y el vidrio molido en cuanto a su reactividad y actividad puzolánica. Se presentan resultados experimentales sobre pastas y morteros con porcentajes variables de ambas adiciones (8, 16 y $24 \%$ en peso). Se analiza la cinética de hidratación temprana por calorimetría de conducción, la potencial puzolanicidad por el ensayo de Frattini y el seguimiento de la hidratación de las pastas por difracción de rayos X (DRX). Se presentan resultados de fluidez y resistencia a compresión sobre morteros normalizados a 2, 7 y 28 días.

Los estudios realizados muestran que la incorporación de vidrio molido como adición, desarrolla más lentamente actividad puzolánica y en menor medida que la MS, sin embargo no reduce la fluidez de los morteros y contribuye significativamente a la resistencia mecánica.

Palabras clave: vidrio molido, puzolanas, hidratación, DRX.

\begin{abstract}
Due their high content of silica in an amorphous state the glass, in theory, can behave as pozzolanic material when are finely ground. This opens up an interesting way of reusing these materials in the Portland cement industry (CP), especially considering that solid urban waste (RSU) in Argentina contains between 3 and 5\% by weight of glass.

This paper studies the behavior of ground white glass from bottles, in compares that corresponding to microsilice (MS), with recognized activity. The objective of this paper is analyzed the structural differences, mineralogical composition, size and particle shape of the MS and the ground glass in terms of reactivity and pozzolanic activity. Experimental results on pastas and mortars with variable percentages of both additions $(8,16$ and $24 \%$ ) are presented. The kinetics of early hydration by conduction calorimetry, the potential pozzolanity by the Frattini test and the X-ray diffraction (DXR) paste hydration monitoring are analyzed. Fluidity and compressive strength results are shown on standar mortars at 2, 7 and 28 days.

In general the incorporation of ground glass develops its reactivity more slowly and to a lesser extent than the MS, however it does not reduce the fluidity of the mortars and contributes significantly to the compressive strength.
\end{abstract}

Key words: ground glass, pozzolans, hydration, DRX. 


\section{INTRODUCCIÓN}

Cada año, millones de toneladas de vidrio son descartadas en el mundo. El vidrio es un material $100 \%$ reciclable que no pierde sus propiedades físicas ni disminuye su calidad en el proceso de reciclado [1]. Por cada tonelada de vidrio que se recicla, se ahorran 130 kilos de combustible y 1.200 kilos de materia prima. Sin embargo es muy bajo el porcentaje de los mismos que se reciclan y se incorporan nuevamente al circuito.

Los vidrios en la composición promedio de los RSU de la zona metropolitana de Buenos Aires, Argentina se encuentran entre el 5,00\% (en 2001) y el 3,09\% (en 2011) [2]. En 2005, aproximadamente 12,8 millones de toneladas de vidrio fueron vertidos en los Estados Unidos [3]. En Hong Kong, de las $64.000 \mathrm{t}$ de residuos de vidrio/ año que se generaron en 2002, sólo unas 8.000 t fueron recicladas y reutilizadas, aunque alrededor de $50.000 \mathrm{t}$ de vidrio residual eran técnicamente recuperables [4]. Pereira- de Oliveira ha reportado para Portugal en 2001, sobre una generación de residuos de vidrio de 493.000 t, que sólo el 25\% de este fue reciclado [5], aunque este valor se duplicó para 2008.

El aprovechamiento de materiales residuales y en especial de vidrios en la industria de la construcción es una atractiva opción. Una interesante aplicación implica el reemplazo parcial de clinker por materiales residuales de composición química y mineralógica compatible. Por su alto contenido de sílice en estado amorfo, los vidrios en teoría pueden comportarse como materiales puzolánicos cuando se muelen finamente [6], lo que abre una interesante vía de reutilización de estos residuos. Las puzolanas se definen como materiales sílico ó sílico-aluminosos los cuales finamente molidos y en presencia de humedad reaccionan con el hidróxido de calcio a temperatura ambiente, formando compuestos con propiedades cementantes [7].

Las puzolanas naturales han sido usadas desde edades antiquísimas para la producción de materiales durables. Cenizas volantes y humos de sílice, son comúnmente los elegidos para la obtención de hormigones resistentes y durables. La sílice amorfa presente en las puzolanas, reacciona con el hidróxido de calcio $(\mathrm{CH})$ generado durante la hidratación de los silicatos de calcio $\left(\mathrm{C}_{3} \mathrm{~S} / \mathrm{C}_{2} \mathrm{~S}\right)$ del $\mathrm{CP}$, formando fases hidratadas similares a los productos de hidratación de los silicatos (C-S-Hn, gel) del CP [8].

Las reacciones mencionadas son del tipo:

$$
\begin{aligned}
& \mathrm{C}_{3} \mathrm{~S} / \mathrm{C}_{2} \mathrm{~S}+\mathrm{nH}_{2} \mathrm{O} \rightarrow \mathrm{C}-\mathrm{S}-\mathrm{H}_{n}+2 \mathrm{CH} \\
& \mathrm{CH}+\text { puzolana } \rightarrow \mathrm{C}-\mathrm{S}-\mathrm{H}_{n}
\end{aligned}
$$

El aprovechamiento de cualquier subproducto industrial y/o material residual tiene en la actualidad gran impacto. La industria cementera juega en este aspecto un rol decisivo, ya que contribuye a la reducción de los desechos, en distintas etapas de la producción, y concretamente en lo que respecta a la incorporación de materiales puzolánicos, con la ventaja adicional de mejorar la calidad del producto final y a menor costo.

Estudios previos han demostrado que el uso de vidrio molido como adición activa a hormigones provee beneficios económicos y ambientales [9], incluso si se incorpora como agregado fino en morteros de cemento blanco [10]. Carsana et al. [11] han reportado que morteros con vidrio molido sumergidos en agua por siete años no han mostrado signos de deterioro y han aumentado su resistencia a compresión. En lo referente al alto contenido de álcalis que poseen los vidrios, Schwarz et al. [12] han informado que el polvo de vidrio libera sólo una fracción muy pequeña de sodio en la solución.

Este trabajo estudia el comportamiento de residuos de vidrio blanco molido procedente de botellas en comparación al correspondiente a la microsílice (MS), de reconocida actividad. El objetivo de este trabajo es analizar las diferencias estructurales, composición mineralógica, y forma de partícula de la MS y el vidrio molido en cuanto a su reactividad y actividad puzolánica. Se presentan resultados experimentales sobre pastas y morteros con porcentajes variables de ambas adiciones $(8,16$ y $24 \%)$. Se analiza la potencial puzolanidad por el ensayo de Frattini, la cinética de hidratación temprana por calorimetría de conducción y el seguimiento de la hidratación de pastas por difracción de rayos X (DRX). Se muestran resultados de fluidez y resistencia a compresión sobre morteros normalizados a 2, 7 y 28 días.

\section{MATERIALES Y MÉTODOS}

Para este estudio se utilizó un cemento portland normal (CP). La composición química del mismo fue obtenida por fluorescencia de rayos X y su composición potencial fue calculada a partir de las ecuaciones de Bogue.

Como adiciones se utilizaron un residuo de vidrio blanco de botella (BG) y microsílice comercial (MS). La composición mineralógica de estos materiales fue obtenida por difracción de rayos X (DRX) y espectroscopía infrarroja (FT-IR), para lo cual se utilizó un equipo Philips X’Pert PW 3710, con ánodo de co- 
bre y monocromador de grafito operando a $40 \mathrm{kV}$ y $20 \mathrm{~mA}$ y un equipo Nicolet Magna 500, dispersando las muestras en $\mathrm{KBr}$, respectivamente. Las micrografías fueron obtenidas utilizando un Microscopio Electrónico de Barrido (SEM) Carl Zeiss EVO-Ma-10.

En este estudio el residuo de vidrio (BG) fue molido de modo que el retenido sobre tamiz $45 \mu \mathrm{m}$ (\#325) fuera menor al 12\%. Para este objetivo se utilizó un molino de bolas de laboratorio con una relación cuerpos moledores/material preestablecida. Al igual que la MS, el BG se usó como reemplazo parcial del CP en 8,16 y $24 \%$ en peso. Las muestras se denominan $8 \mathrm{MS}, 16 \mathrm{MS}$ y $24 \mathrm{MS}$; $8 \mathrm{BG}, 16 \mathrm{BG}$ y $24 \mathrm{BG}$.

El seguimiento de la hidratación temprana (primeras 48 horas) fue realizado por calorimetría isotérmica de conducción y posteriormente por DRX, sobre pastas con diferentes porcentajes de reemplazo.

La resistencia mecánica a la compresión de los cementos mezcla y el patrón se midió sobre morteros normalizados a 2, 7 y 28 días. La relación a/c se fijó en 0.5, sobre estos morteros se midió la fluidez, los resultados se informaron en porcentaje respecto a la referencia sin adición. La puzolanicidad de cada mezcla fue evaluada mediante el ensayo de Frattini a las edades de 2, 7 y 28 días

\section{RESULTADOS Y DISCUSIÓN}

\subsection{Materias primas}

La composición química del CP, se muestra en la Tabla 1. La composición potencial del mismo fue: $\mathrm{C}_{3} \mathrm{~S}$ : $60 \%, \mathrm{C}_{2} \mathrm{~S}: 16.4 \%, \mathrm{C}_{3} \mathrm{~A}: 3.8 \%$ y $\mathrm{C}_{4} \mathrm{AF}: 11.5 \%$. La superficie específica Blaine fue de $315 \mathrm{~m}^{2} / \mathrm{kg}$.

Tabla 1: Composición química del cemento portland (\%).

\begin{tabular}{|l|l|l|l|l|l|l|l|l|}
\hline $\mathrm{SiO}_{2}$ & $\mathrm{Al}_{2} \mathrm{O}_{3}$ & $\mathrm{Fe}_{2} \mathrm{O}_{3}$ & $\mathrm{CaO}$ & $\mathrm{MgO}$ & $\mathrm{SO}_{3}$ & $\mathrm{Na}_{2} \mathrm{O}$ & $\mathrm{K}_{2} \mathrm{O}$ & $\mathrm{PPC}$ \\
\hline 21.5 & 3.8 & 3.8 & 64.3 & 0.8 & 2.6 & 0.1 & 1.1 & 2.1 \\
\hline
\end{tabular}

La composición mineralógica de las adiciones BG y MS fueron obtenidas por difracción de rayos X (DRX) y espectroscopia infrarroja (FT-IR). Los resultados se muestran en la Figura 1. El estado amorfo del BG y la MS se observa claramente por DRX. No se identifican picos que puedan atribuirse a fases cristalinas y sólo puede identificarse una ancha banda difusa entre $15-40^{\circ}$ de $2 \theta$, asignada a la fase amorfa [6,13].

Por FT-IR, la asignación se realiza en base al tetraedro $\mathrm{SiO}_{4}{ }^{4-}$, el cual presenta en este caso una simetría menor a Td. La vibración de estiramiento $\mathrm{Si}-\mathrm{O}-\mathrm{Si}$ se encuentra en la región entre $700-1200 \mathrm{~cm}^{-1}$ acoplada con otros vibradores de la estructura. Las vibraciones de deformación O-Si-O se encuentran entre 400-500 $\mathrm{cm}^{-1}[14]$.
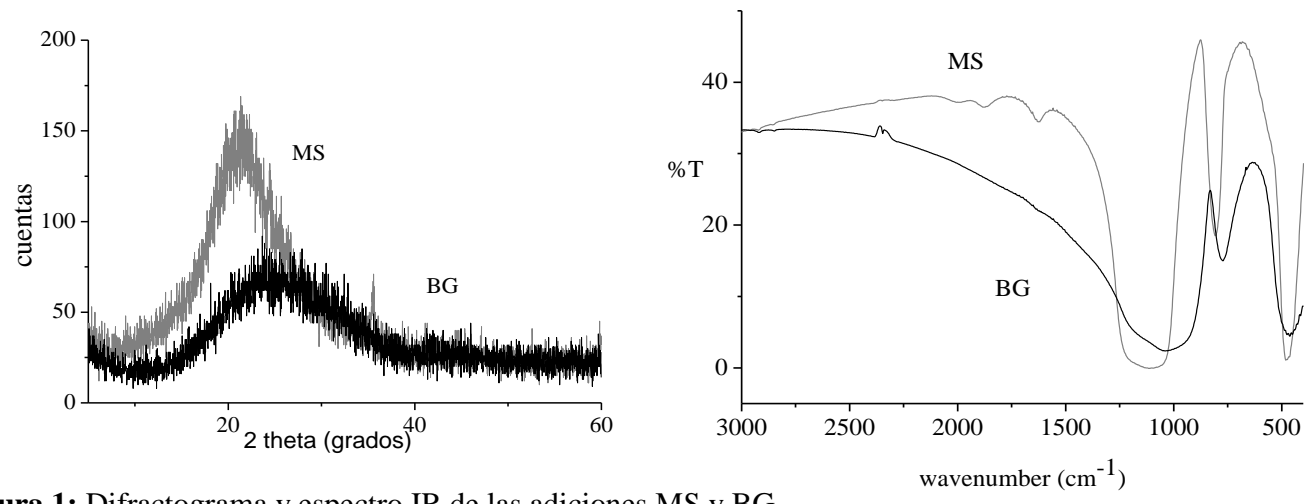

Figura 1: Difractograma y espectro IR de las adiciones MS y BG

En cuanto a las características físicas de los materiales usados, en general la bibliografía considera necesario que las adiciones posean partículas más finas que las del cemento que reemplaza, Khmiri et al. [15] recomiendan partículas menores a $20 \mu \mathrm{m}$ para obtener muy buena actividad puzolánica y partículas menores a $40 \mu \mathrm{m}$ para obtener buena actividad puzolánica. En coincidencia, Federico et al. [16] postulan que por debajo de $45 \mu \mathrm{m}$ las partículas de vidrio pueden ser puzolánicas. En consecuencia, la consigna de molienda 
significó determinar cada 30 minutos la curva granulométrica y sus parámetros $\left(\mathrm{d}_{90}, \mathrm{~d}_{50}\right.$ y d $\left.\mathrm{d}_{10}\right)$. Cuando el $\mathrm{d}_{90}$ fue menor a $45 \mu \mathrm{m}$ se dio por finalizada la molienda, los valores obtenidos se presentan en la Tabla 2 .

Tabla 2: Característica de los materiales

\begin{tabular}{|l|l|l|l|l|}
\hline MATERIAL & $\mathbf{d}_{10}, \mu \mathrm{m}$ & $\mathbf{d}_{50}, \mu \mathrm{m}$ & $\mathbf{d}_{90}, \mu \mathrm{m}$ & DENSIDAD $\mathrm{kg} / \mathrm{m}^{3}$ \\
\hline $\mathbf{C P}$ & 2.50 & 18.87 & 63.26 & 3150 \\
\hline MS & 2.95 & 10.05 & 20.36 & 2115 \\
\hline BG & 1.65 & 7.48 & 24.55 & 2571 \\
\hline
\end{tabular}

La Figura 2 muestra las micrografías SEM (scanning electron microscopy) de las adiciones MS y BG, donde se puede observar la diferencia en el tamaño y forma de las partículas. El polvo de vidrio molido consiste principalmente en partículas de forma alargada y/o placas con un gran intervalo de tamaños. La MS tiene una morfología muy diferente, presentando partículas redondeadas y con un tamaño más homogéneo que el polvo de vidrio.
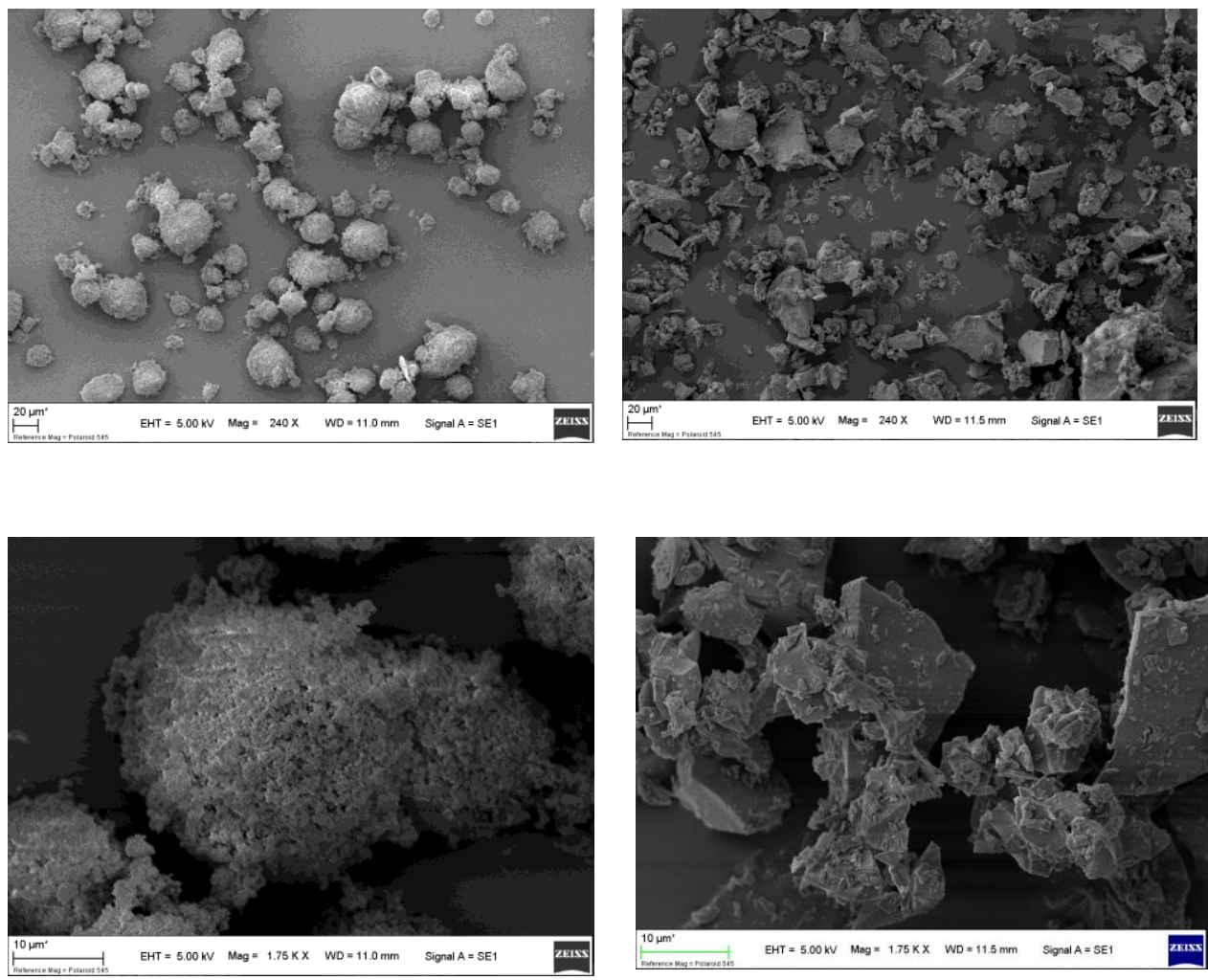

Figura 2: Micrografías SEM aspecto superficial de MS (izquierda) y BG (derecha), 240x y 1.75k x.

\subsection{Hidratación temprana por calorimetría}

En la Figura 3 se presentan las curvas de velocidad de liberación de calor del CP y de los cementos mezcla obtenidas con las adiciones en los diferentes porcentajes de reemplazo estudiados. Se muestra además la curva de calor acumulado (Qt) para el CP.

La incorporación de MS acelera y estimula las reacciones, adelantando la aparición del máximo y aumentando su intensidad. La curva de hidratación revela el efecto de estimulación especialmente del $\mathrm{C}_{3} \mathrm{~A}$ del cemento al desdoblarse el pico principal, ya que el primero corresponde a la hidratación de los silicatos y el segundo a la de los aluminatos [17]. Tal es así que para la muestra 16MS el Qt generado, es el mayor de to- 
das las muestras en las primeras 48 horas de hidratación, indicando la máxima estimulación. La muestra 24MS queda retrasada respecto a 16MS a pesar del mayor porcentaje de adición activa que posee, esto se atribuye al hecho de que todas las pastas fueron hidratadas con igual a/c por lo cual la pasta 24MS por el gran volumen de adición incorporada debió requerir mayor cantidad de agua para el humedecimiento superficial y la hidratación [18].

En general, y como fuera observado por Shi et al. [6] la incorporación de BG genera una disminución del Qt respecto al patrón, una extensión del período durmiente y un ligero corrimiento del tiempo de aparición del máximo. Esta observación es atribuida principalmente al efecto de dilución [19] durante la primera etapa de la hidratación del cemento mezcla. En el caso de la incorporación de diferentes porcentajes de BG se observa claramente la disminución del Qt por efecto de la dilución, pero estos aumentos no afectan la cinética de hidratación.
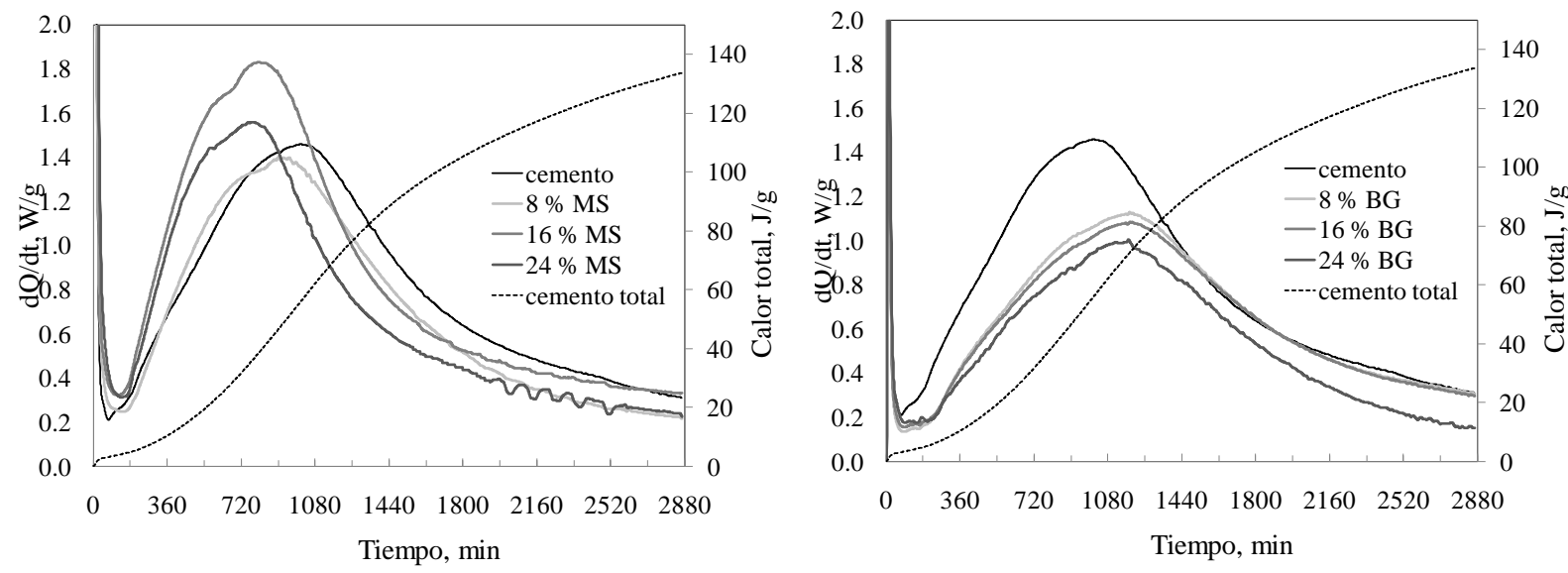

Figura 3: Curvas calorimétricas de $\mathrm{CP}$ y los diferentes cementos mezclas, primeras 48 horas.

\subsection{Seguimiento de la hidratación por DRX}

El estudio del progreso de la hidratación de CP y de los cementos mezclas con diferentes porcentajes de MS y BG se realizó por DRX, sobre muestras hidratadas a diferentes edades. En todos los casos se identificaron las mismas fases principales a la misma edad de hidratación, variando solamente en la intensidad de los picos debido a la presencia o no de adiciones y según el porcentaje de reemplazo realizado.

La Figura 4 muestra en forma comparativa el comportamiento según el avance de la hidratación de CP, 24MS, 24BG a 2, 7 y 28 días de hidratación. Se identifican principalmente ettringita (Et), hidróxido de calcio $(\mathrm{CH})$ y restos de silicatos sin hidratar. En cuanto a la diferencia en intensidad de los principales picos, la más significativa es la disminución de la intensidad del pico de $\mathrm{CH}$ con el avance de la hidratación en las muestras con BG y MS, a diferencia de lo que ocurre con CP dónde se observa un aumento de la intensidad del pico $\mathrm{CH}$ por las reacciones típicas de hidratación del silicato tricálcico $\left(\mathrm{C}_{3} \mathrm{~S}\right)$ y dicálcico $\left(\mathrm{C}_{2} \mathrm{~S}\right)$ del cemento (ecuación 1).
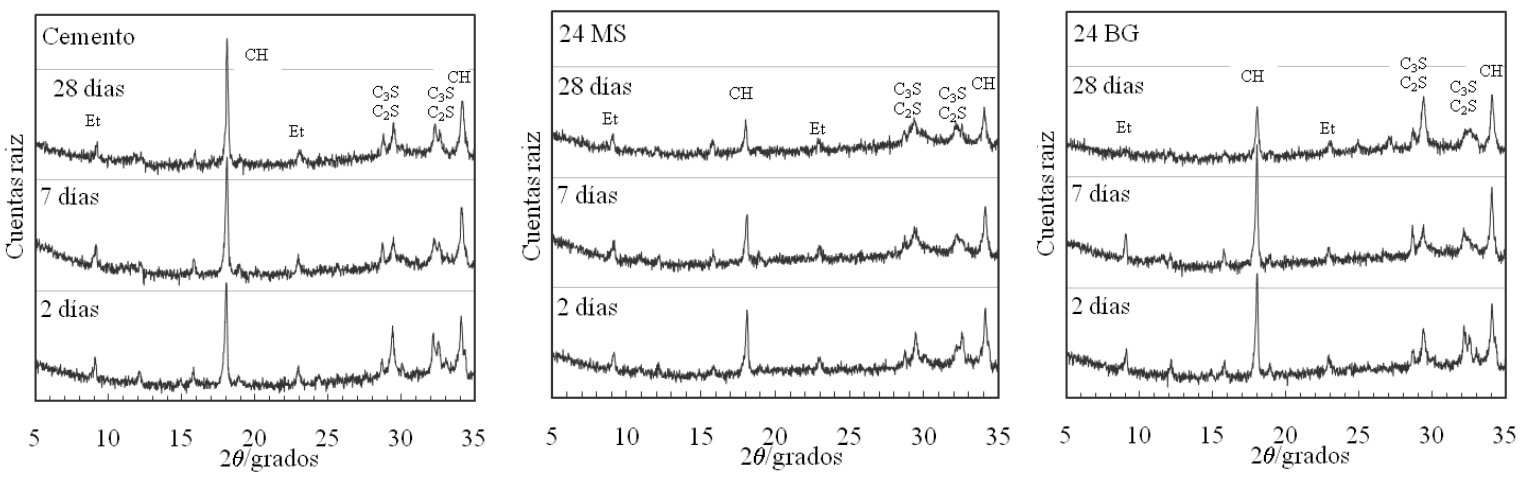

Figura 4: Difractogramas de CP, 24MS y 24BG a 2, 7 y 28 días de hidratación. 
La disminución de la intensidad del pico asignado a $\mathrm{CH}$ a pesar del avance de la hidratación en 24BG y 24MS, se atribuye a la acción puzolánica que como se indicó genera el consumo de $\mathrm{CH}$ por formación de S-C-H adicional con la sílice aportada por la adición (ecuación 2).

El estudio del progreso de la hidratación de los cementos mezclas con diferentes porcentajes de MS y BG se muestran en la figura 5 para la edad de 28 días. Al estudiar el avance de la hidratación en forma comparativa para las dos adiciones y los diferentes porcentajes de reemplazo se observa un mismo comportamiento, sólo que el efecto puzolánico es notablemente mayor cuando el reemplazo es por MS (en todos los porcentajes adicionados) que cuando se usa BG.
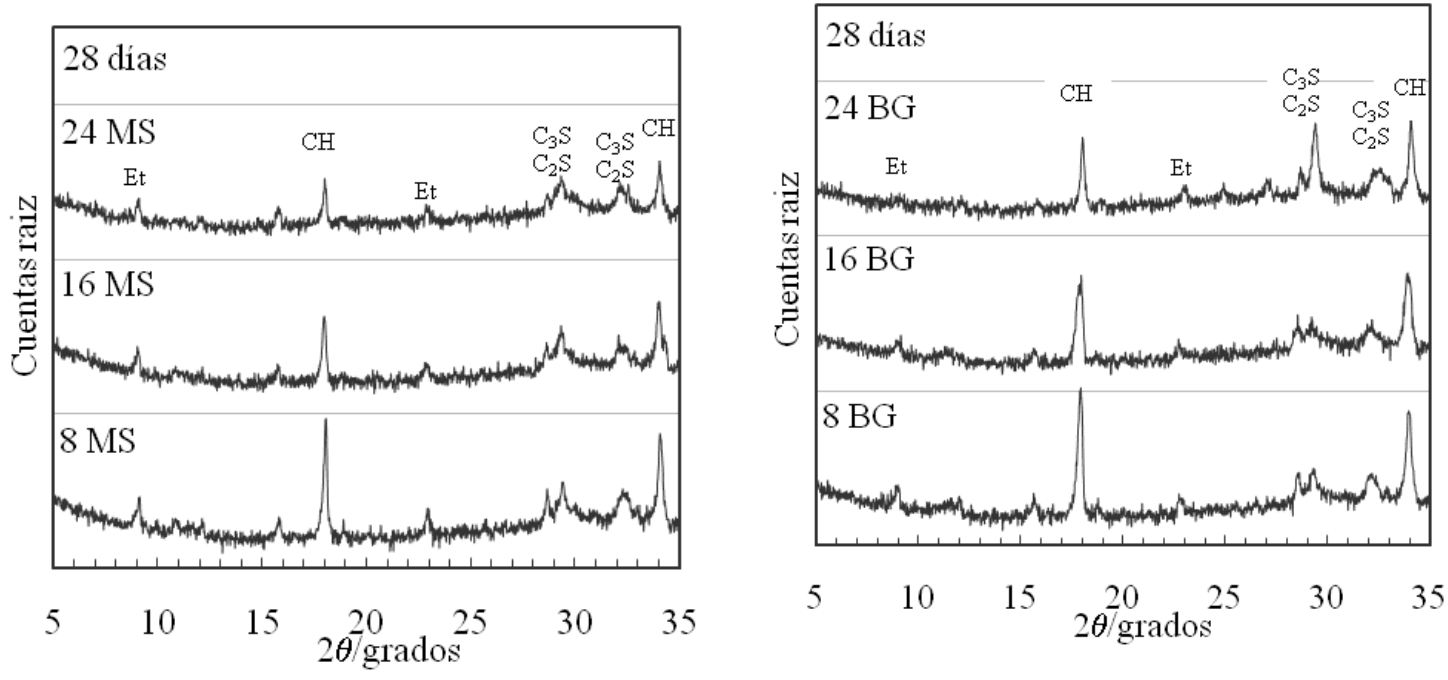

Figura 5: Muestras con MS y BG hidratadas a 28 días, con diferentes porcentajes de adición

\subsection{Fluidez y Resistencia mecánica a la compresión}

En la Tabla 3 se presentan los resultados de fluidez (en \%) de los morteros ensayados. Los mismos se confeccionaron con una relación fija a/c, en consecuencia la muestra 24MS no fue posible moldear por elevada demanda de agua. Por su parte la muestra 8MS provocó una disminución de la fluidez al 70\%. Este comportamiento coincide con el reportado previamente por Rahhal et al. [18] quienes atribuyeron al tamaño y morfología de las partículas la gran absorción de la MS en comparación a otras adiciones. Puede observarse además, que la fluidez de los morteros con la adición de vidrios (8BG y 24BG) son menores que la del mortero patrón. Esta disminución no varía con el aumento del porcentaje de reemplazo.

En general, este comportamiento puede deberse en parte a que las partículas de adición que reemplazan a las de cemento tienen mayor finura que él (ver Tabla 2), necesitando entonces mayor cantidad de agua para poder mojar toda su superficie; y en parte a la menor densidad de las adiciones (ver Tabla 2) dado que como los reemplazos fueron en peso, mayor volumen y cantidad de partículas fueron incorporadas.

Tabla 3: Fluidez (\%) de los morteros

\begin{tabular}{|l|l|l|l|}
\hline PATRÓN & 8MS & 8BG & 24BG \\
\hline $127.5 \%$ & $70 \%$ & $82 \%$ & $82 \%$ \\
\hline
\end{tabular}

La Figura 6 muestra los resultados de resistencia mecánica a la compresión de los morteros de CP y con los diferentes porcentajes de las adiciones estudiadas. A medida que crece la edad, se puede observar que el progreso de la resistencia se mantiene con igual tendencia para todas las muestras. 


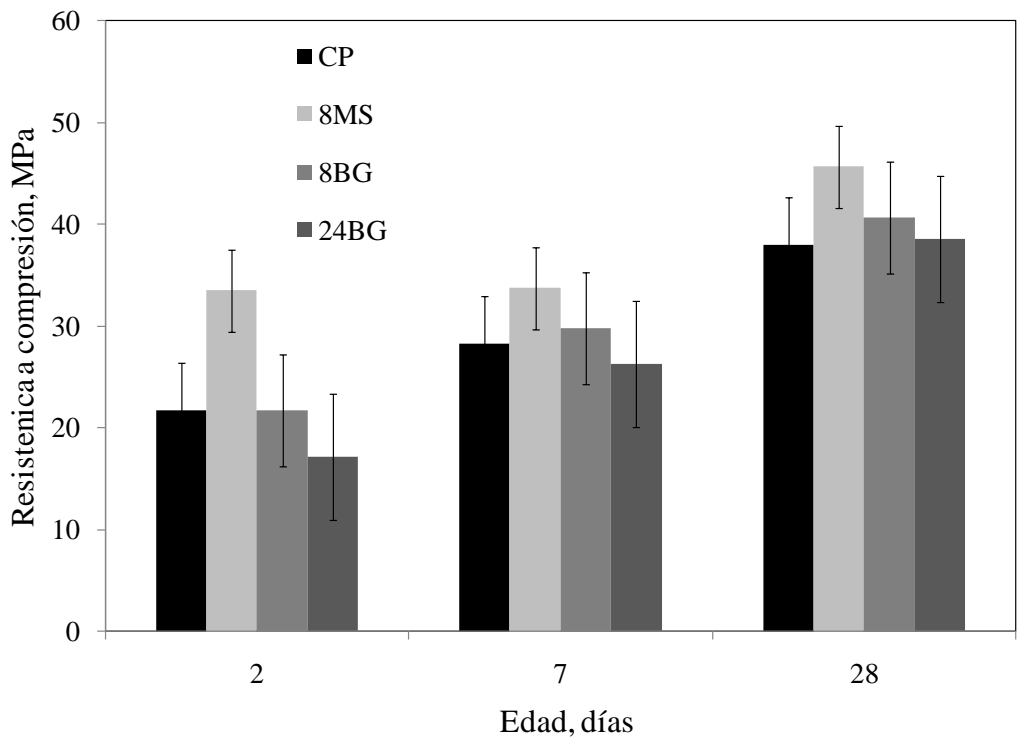

Figura 6: Resistencia a compresión de morteros a 2, 7 y 28 días.

A todas las edades, la resistencia alcanzada por la muestra 8MS supera al patrón alcanzando aproximadamente a 28 días, un $17 \%$ más de resistencia que $\mathrm{CP}$, todo esto a pesar de poseer un $8 \%$ menos de cemento.

Para la muestra 8BG el efecto de estimulación generado por la adición compensa el reemplazo a la edad de 2 días y lo supera a 7 y 28 días, esto es atribuido a la acción puzolánica. Para la muestra 24BG la resistencia lograda a 2 y 7 días no alcanza el valor correspondiente al patrón, pero lo supera levemente a 28 días a pesar del alto porcentaje de reemplazo, mostrando nuevamente actividad puzolánica. Estos resultados están en coincidencia con lo reportado por Schwarz et al. [12], quienes obtuvieron índices de actividad puzolánica determinados por resistencia a compresión, superiores a 0.80 con reemplazos del $20 \%$ en peso.

\subsection{Actividad Puzolánica}

La Figura 7 muestra los resultados de puzolanicidad de los cementos mezclas obtenidos por medio del ensayo normalizado de Frattini. Este ensayo evalúa la actividad puzolánica a partir de la reacción entre el material adicionado y el $\mathrm{Ca}(\mathrm{OH})_{2}$ generado por la hidratación del $\mathrm{CP}$, el resultado se considera positivo si los puntos caen por debajo de la curva de solubilidad. Las muestras ensayadas contenían 8, 16 y $24 \%$ de reemplazo por MS y BG y se ensayaron a las edades de 2, 7 y 28 días.

Las muestras con MS (a excepción de 8MS a 2 días), dieron positivo el ensayo de Frattini a todas las edades de ensayo. La actividad puzolánica mostrada por la MS, revela su alta reactividad al disminuir simultáneamente la $[\mathrm{CaO}]$ y la $\left[\mathrm{OH}^{-}\right]$.

En el caso de BG a la edad de 2 días se observa estimulación por resultar los valores de la [CaO] y la $\left[\mathrm{OH}^{-}\right]$, del mismo orden a pesar del aumento del porcentaje de reemplazo. Con el tiempo y en todas las muestras se observa una disminución de la $[\mathrm{CaO}]$ atribuidas al consumo generado por la reacción puzolánica (ecuación 2). El ensayo da positivo para 24BG a los 7 días y para 8 y 16 BG a edades mayores a 7 días.

El crecimiento de la $\left[\mathrm{OH}^{-}\right]$con el porcentaje de reemplazo y con la edad, se atribuye al aporte de álcalis que genera la adición. La cantidad y cinética de remoción de álcalis en este tipo de cementos mezcla ha sido estudiado por Schwarz et al. [12], informando que sólo una pequeña fracción es disuelta en el agua de poro. Esa cantidad si bien modifica el $\mathrm{pH}$ es insuficiente para acelerar el fraguado (como muestran las curvas de la figura 3) o incrementar la resistencia temprana (como se observa en los morteros a la edad de 2 días, figura 6). 


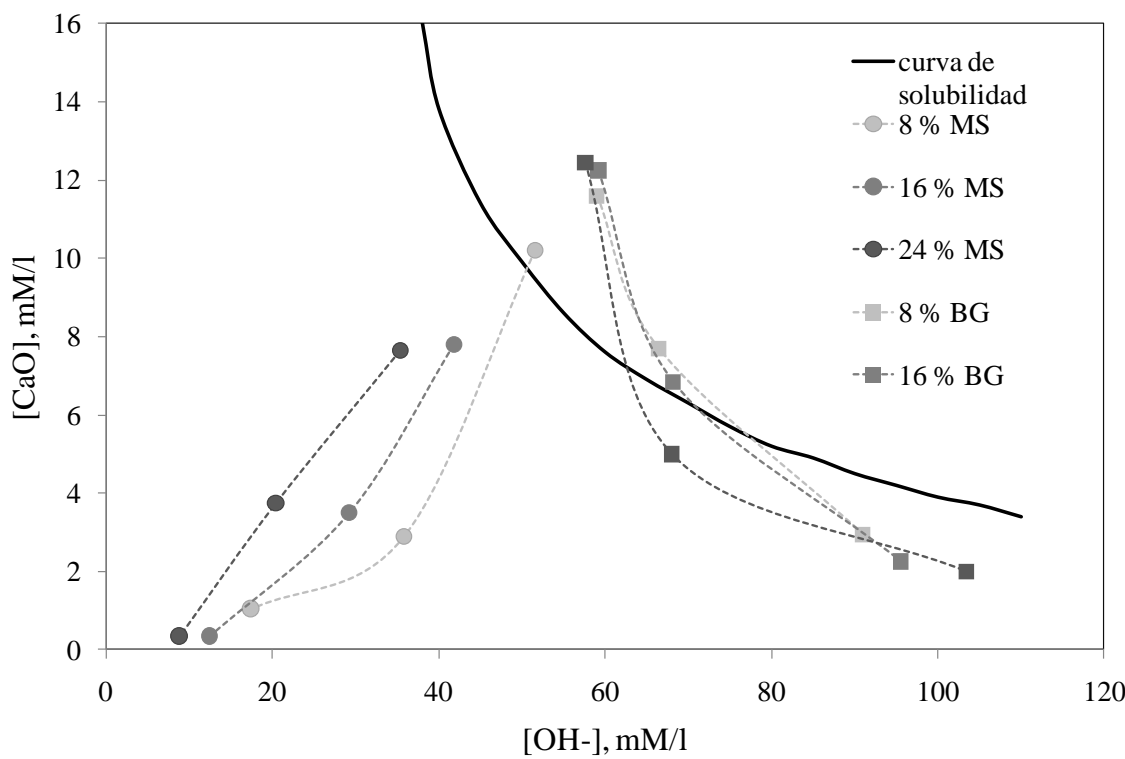

Figura 7: Ensayo de Frattini a 2, 7 y 28 días de hidratación

\section{CONCLUSIONES}

Este estudio compara el comportamiento de residuos de vidrio blanco molido (BG) procedente de botellas con el correspondiente a la microsílice (MS), utilizada habitualmente como adición activa al CP y permitió concluir:

La caracterización química y mineralógica de ambas adiciones no presenta grandes diferencias, tratándose básicamente de sílice amorfa.

Luego de la molienda, las partículas de BG observadas por micrografías SEM mostraron diferencias especialmente en su forma. El polvo de vidrio molido consiste principalmente en partículas de forma alargada y/o placas mientras que la MS presenta partículas redondeadas y con un tamaño homogéneo.

Con respecto a la hidratación temprana, la incorporación de vidrio molido al cemento portland, ejerce en principio efecto de dilución retrasando las reacciones de hidratación, alargando el período durmiente y disminuyendo la cantidad de calor liberada a edades tempranas. Por su parte la MS estimula las reacciones de hidratación acortando el período durmiente y elevando el calor de hidratación.

Con el avance de la hidratación BG muestra actividad puzolánica, esto lleva a un aumento relativo de la resistencia que permite a medida que avanza la hidratación cubrir el efecto de dilución generado por el reemplazo. En el caso de la MS presenta una elevada reactividad, y en consecuencia un significativo aumento de la resistencia con respecto al patrón.

El ensayo de Frattini, confirmó que ambas adiciones son puzolánicas, aunque MS se muestra siempre más reactiva que $\mathrm{BG}$, además la $\left[\mathrm{OH}^{-}\right]$reveló baja alcalinidad para las muestras con MS y elevada para las muestras con BG.

La utilización de BG como adición activa al CP es posible aunque su reactividad es menor a toda edad y esta retrasada en el tiempo respecto a la MS.

\section{AGRADECIMIENTOS}

Los autores agradecen el apoyo financiero recibido de la Facultad de Ingeniería (UNCPBA) y CIFICEN (UNCPBA, CONICET, CIC).

\section{BIBLIOGRAFÍA}

[1] PALOMO T.V., "Reciclar vidrio es ahorrar combustible", http://www.justoysustentable.org/2012/04/reciclar-vidrio-es-ahorrar-combustible.html. Accedido en junio de 2016.

[2] CEAMSE, Ecología urbana. http://www.ceamse.gov.ar/wp-content/uploads/2012/06/Tercer-InformeECRSU-AMBA1.pdf. Accedido en diciembre de 2016. 
[3] MIRZAHOSSEINI, M., RIDING, K. "Effect of curing temperature and glass type on the pozzolanic reactivity of glass powder", Cement and Concrete Research, v. 58, pp. 103-111, April 2014.

[4] CHEN G., LEE, H., YOUNG, K.L., et al., "Glass recycling in cement production-an innovative approach", Waste Management, v. 22, n- 7, pp. 747-753, November 2002.

[5] PEREIRA-DE-OLIVEIRA, L.A., GOMES, J.P.G., SANTOS, P.M.S., et al., "The potential pozzolanic activity of glass and red-clay ceramic waste as cement mortars components", Construction and Building Materials, v. 31, pp. 197-203, June 2012.

[6] SHI C., ZHENG K. "A review on the use of waste glasses in the production of cement and concrete", Resources, Conservation and Recycling, v. 52, n. 2, pp 234-247, December 2007.

[7] ASTM C618-12a: Standard Specification for Fly Ash and Raw or Calcined Natural Pozzolan for use as a Mineral Admixture in Portland Cement Concrete

[8] SABIR, B.B., WILD, S., BAI, J., et al., "Metakaolin and calcined clays as pozzolans for concrete: a review", Cement and Concrete Composites, v. 23, n. 6, pp. 441-454, December 2001.

[9] MARAGHECHI, H., MARAGHECHI, M., RAJABIPOUR, F., et al., "Pozzolanic reactivity of recycled glass powder at elevated temperatures: Reaction stoichiometry, reaction products and effect of alkali activation", Cement \& Concrete Composites, v.53, pp. 105-114, October 2014.

[10] LING, T-Ch., POON, C.S., KOU, S.C., et al., "Feasibility of using recycled glass in architectural cement mortars", Cement \& Concrete Composites, v. 33, n. 8, pp 848-854, September 2011.

[11] CARSANA, M., FRASSONI, M., BERTOLINI, L., et al., "Comparison of ground waste glass with other supplementary cementitious materials", Cement \& Concrete Composites, v. 45, pp.39-45, January 2014.

[12] SCHWARZ, N., NEITHALATH, N., "Influence of a fine glass powder on cement hydration: Comparison to fly ash and modeling the degree of hydration", Cement and Concrete Research, v.38, n.4, pp. 429436, April 2008.

[13] SOLIMAN, N.A., TAGNIT-HAMOU, A. "Partial substitution of silica fume with fine glass powder in UHPC: Filling the micro gap", Construction and Building Materials, v. 139, pp. 374-383, May 2017.

[14] VILLAQUIRÁN-CAICEDO M. A., et al., "Microstructure Assessment of Metakaolin BasedGeopolymers Producedwith Alternative Silica Sources Exposed to High Temperaturas", Ingeniería Investigación y Tecnología, v. XVI, n.1, pp. 113-122, enero-marzo 2015.

[15] KHMIRI A., CHAABOUNI, M., SAMET, B., et al., "Chemical behavior of ground waste glass when used as partial cement replacement in mortars", Construction and Building Materials, v. 44, pp. 74-80, July 2013.

[16] FEDERICO, L.M., CHIDIC, S.E., "Waste glass as a supplementary cementitious material in concrete Critical review of treatment methods", Cement \& Concrete Composites, v. 31, n.8, pp 606-610, September 2009.

[17] MINDESS, S., YOUNG, J.F. Concrete, cap.4. USA, Prentice-Hall Inc., 1981.

[18] RAHHAL, V.F., TALERO, R. "Calorimetry of Portland cement with silica fume, diatomite and quartz additions", Construction and Building Materials, v. 23, pp. 3367-3374, November 2009.

[19] CYR M., LAWRENCE, P., RINGOT, E., et al., "Mineral admixtures in mortars: quantification of the physical effects of inert materials on short-term hydration", Cement and Concrete Research, v. 35, pp.719730, April 2005. 